\title{
Opioid Antagonists, Partial Agonists, and Agonists/Antagonists: The Role of Office- Based Detoxification
}

Standiford Helm II', MD, Andrea M. Trescot², MD, James Colson ${ }^{3}, \mathrm{MD}$, Nalini Sehgal ${ }^{4}, \mathrm{MD}$, and Sanford Silverman ${ }^{5}, \mathrm{MD}$

From: ${ }^{1}$ Pacific Coast Pain Management Center, Laguna Hills, CA

${ }^{2}$ University of Florida, Gainesville, FL; ${ }^{3}$ West Virginia University Hospitals, Morgantown, WV; ${ }^{4}$ University of Wisconsin School of Medicine and Public Health, Madison, WI; and ${ }^{5}$ Comprehensive Pain

Management, Pompano Beach, FL.

Dr. Helm is Medical Director, Pacific Coast Pain Management Center, Laguna Hills, CA. Dr. Trescot is Director of the Pain Program at the University of Florida and the Malcolm Randall VA Medical Center, Gainesville, FL.

Dr. Colson is Assistant Professor of Anesthesiology, Department of Anesthesiology, Pain Medicine Service,

West Virginia University Hospitals, Morgantown, WV.

Dr. Sehgal is the Director of the Interventional Pain Program at the University of Wisconsin School of Medicine and Public Health and Associate Professor

Rehabilitation Medicine, Madison, WI.

Dr. Silverman is the Medical Director, Comprehensive Pain Management, Pompano Beach, FL.

Address Correspondence: Standiford Helm, MD

Pacific Coast Pain Management Center 24902 Moulton Parkway Suite 200

Laguna Hills, Ca 92637 e-mail: Drhelm@pcpmc.com

Funding: Internal funding was provided by the American Society of Interventional Pain

Physicians limited to travel and lodging expenses of the authors. Conflict of Interest: None.

Free Full Manuscript: www.painphysicianjournal.com
Background: The opioid receptor antagonists naloxone and naltrexone are competitive antagonists at the mu, kappa, and sigma receptors with a higher affinity for the mu receptor and lacking any mu receptor efficacy. Buprenorphine is classified as a partial agonist. It has a high affinity, but low efficacy at the mu receptor where it yields a partial effect upon binding. It also, however, possesses kappa receptor antagonist activity making it useful not only as an analgesic, but also in opioid abuse deterrence, detoxification, and maintenance therapies. Naloxone is added to sublingual buprenorphine (Suboxone $\AA$ ) to prevent the intravenous abuse of buprenorphine. The same product (sublingual buprenorphine) when used alone (i.e. without naloxone) is marketed as Subutex®.

Objective: To evaluate and update the available evidence regarding the use of agonist/antagonists to provide office-based opioid treatment for addiction.

Methods: A review using databases of EMBASE and MEDLINE (1992 to December 2007). These included systematic reviews, narrative reviews, prospective and retrospective studies, as well as cross-references from other articles.

Outcome Measures: The primary outcome measure was treatment retention. Other outcome measures included opioid-free urine drug testing, opioid craving, intensity of withdrawal, pain reduction, adverse effects, addiction severity index, and HIV risk behavior.

Results: The results found 17 studies, 1 systematic review, 12 RCTs, and 4 observational series, which document the efficacy and safety of buprenorphine alone and in combination with naloxone in detoxifying and maintaining abstinence from illicit drugs in patients with opioid addiction.

Conclusion: Based on the present evaluation, it appears that opioid antagonists, partial agonists, and antagonists are useful in office-based opioid treatment for addiction.

Key words: Opioid, antagonist, partial agonist, tolerance, dependence, detoxification, withdrawal, hyperalgesia, buprenorphine, naloxone, naltrexone, methylnaltrexone, butorphanol, nalbuphine, pentazocine

Pain Physician 2008; 11:225-235 


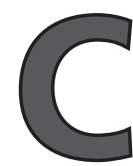

ompounds can have intrinsic affinity and efficacy at receptors, with affinity being a measure of the "strength of interaction" between a compound binding to its receptor and efficacy being a measure of the strength of activity or effect from this binding at the receptor. An agonist has both affinity and efficacy; an antagonist has affinity but no efficacy; a partial agonist/antagonist has affinity, but only partial efficacy. Regarding the opioids, the relevant receptors are the mu, kappa, and delta receptors. Compounds can have differing degrees of affinity and efficacy at these various receptors.

The opioid receptor antagonists naloxone and naltrexone are competitive antagonists at the mu, kappa, and sigma receptors with a higher affinity for the mu receptor and lacking any mu receptor efficacy. Naloxone and naltrexone act centrally and peripherally, but have differing pharmacokinetic profiles favoring different therapeutic uses. Naloxone has low oral bioavailability, but a fast onset of action following parenteral administration for rapid reversal of acute adverse opioid effects. Its short duration of action risks the potential for "re-narcotization," thus not providing adequate duration of effect coverage for opioid maintenance or deterrent therapy. Naltrexone is orally effective with a long duration of action making it useful in abuse deterrent, detoxification, and maintenance treatment modalities. Nalmefene, a mu opioid receptor antagonist, is a water-soluble naltrexone derivative with a longer duration of action than naloxone. Naloxone and naltrexone can be combined with mu agonists or partial agonists. Ultralow dose naltrexone combined with oxycodone (Oxytrex $\left.{ }^{\circledR}\right)$ is currently under study to see if the naltrexone will suppress opioid tolerance. Nalmefene is available for use in the United States for the reversal of opioid drug effects (1). Methylnaltrexone and alvimopan are peripherally acting mu receptor antagonists currently under investigation for use in treating postoperative ileus and opioid-induced bowel dysfunction.

Buprenorphine is classified as a partial agonist. It has a high affinity, but low efficacy at the mu receptor where it yields a partial effect upon binding. It also, however, possesses kappa receptor antagonist activity making it useful not only as an analgesic, but also in opioid abuse deterrence, detoxification, and maintenance therapies. Buprenorphine (Buprenex ${ }^{\circledR}$ ) has been approved for use in the United States since December 1981. A 72-hour transdermal product designed to continuously release buprenorphine at $35,52.5$, or
$70 \mathrm{mcg} / \mathrm{hr}$ is available in Europe (but not in the United States) for the treatment of persistent pain. Naloxone is added to sublingual buprenorphine (Suboxone ${ }^{\circledR}$ ) to prevent the intravenous abuse of buprenorphine. The same product (sublingual buprenorphine) when used alone (i.e., without naloxone) is marketed as Subutex®.

Buprenorphine has a poor bioavailability with extensive first pass effect by the liver. Conversely, because of high lipid solubility, it has an excellent sublingual bioavailability. The typical daily dose for opioid addiction ranges from 4 to $32 \mathrm{mg}$ daily. The naloxone component exhibits almost no sublingual absorption and very little oral absorption. The intent of its addition is to reverse the effects of an IV or IM administered buprenorphine. After sublingual administration there is a rapid onset of effect ( $30-60$ minutes) with a peak effect at about $90-100$ minutes. It is used on a once-a-day dose for maintenance therapy. Buprenorphine is primarily metabolized by P450 3A4. There are extensive drug-drug interactions which can exist, based on the induction or inhibition of the 3A4 system. Buprenorphine's usual adverse effects may include sedation, nausea and/or vomiting, dizziness, headache, and respiratory depression.

Partial agonist-antagonists, such as nalorphine, pentazocine, nalbuphine, and butorphanol, have high mu affinity but have poor mu efficacy and also have kappa agonist activity. These agents can be used as analgesics, but have partial or a ceiling to their analgesic effect, such that escalating the dosage beyond a certain level will only yield greater opioid side effects. The stimulation of kappa receptors can provide undesired dysesthesia, as with Talwin $®$.

A unique analgesic, Tramadol, is an atypical opioid, a 4-phenyl-piperidine analogue of codeine, with partial mu agonist activity in addition to central GABA, catecholamine and serotonergic actvities. Tramadol is used primarily as an analgesic, but has demonstrated usefulness in treating opioid withdrawal (2).

\section{Office-Based Opioid Treatment (OBOT)}

The most important use currently for this class of medications is office-based opioid detoxification/ treatment. With the current trends in prescription opioid abuse and the overlap of pain and addiction, it behooves the pain specialist to be aware of the current treatments for opioid addiction.

Historically, opioid dependence has been a problem for most of the twentieth century. Before the 
Harrison Narcotic Act of 1914 was enacted, physicians could prescribe opioids for any condition, including opioid dependence. In 1919, the US Supreme Court ruled that the Harrison Act disallowed prescription of opioids for maintenance purposes, which effectively ended OBOT for addiction.

After World War I, many cities established maintenance clinics for opioid addiction to respond to a huge wave of heroin addicts. In New York City, pioneering efforts were engaged in the treatment of more than 8,000 addicts through the city's health department. Unfortunately, these clinics were shut down by the federal government. From the 1920s forward, physicians were discouraged from treating opioid addiction, and it was reconceptualized as a criminal rather than a medical problem (1).

It was not until the 1970s when opioid addiction was addressed at the federal level with methadone regulations (21 CFR Part 291) in 1972 and the Narcotic Addict Treatment Act of 1974 which created federal and state licensed methadone clinics. A physician who wished (until very recently) to treat opioid addiction with methadone had to obtain additional registration from the DEA and DHHS with additional approval from state authorities, thus involving an intimidating beaurocratic gauntlet that few physicians have been willing to negotiate.

In October 2002, the US FDA approved Schedule III sublingual buprenorphine tablets for the use in treatment of opioid addiction. The Drug Addiction Treatment Act (DATA) of 2000, an amendment to the Controlled Substances Act, allowed certified physicians to prescribe and dispense Schedule III, IV, and V narcotic drugs that have been approved by the Food and Drug Administration for use in addiction treatment (i.e., maintenance or medical withdrawal/detoxification). Physicians who wish to prescribe buprenorphine for the treatment of opioid dependence must meet 1 or more of the following requirements:

- Board certified in Addiction Psychiatry

- Certified in Addiction Medicine by American Society of Addiction Medicine (ASAM)

- Certified in Addiction Medicine by American Osteopathic Association (AOA)

- Investigator in buprenorphine clinical trials

- Has completed 8 hours of CME provided by American Psychiatric Association, American Academy of Addiction Psychiatry, ASAM, American Medical
Association, AOA (or other organizations designated by Health and Human Services)

- Training/experience as determined by State licensing board

- Other criteria established by Health and Human Services

Qualified physicians must submit notification to Center for Substance Abuse Treatment (CSAT) and a unique identification is then issued from the DEA in the form of an " $X$ " number, thus giving these physicians 2 different DEA numbers. Initially, a provider was limited to treating no more than 30 patients, but in 2007 this was increased to 100 with secondary notification to CSAT.

Buprenorphine is commercially available for pain control in an injectable form known as Buprenex®. It should be noted that the only legally approved form of buprenorphine for the treatment of opioid dependence is the sublingual form (Subutex $₫$, Suboxone $₫$ ).

Prior to its use in the United States, buprenorphine in its sublingual form (Subutex $\AA^{\circledR}$ ) was used very successfully in Europe for the treatment of opioid addiction. Over 10 years of clinical research has supported the use of buprenorphine and its combination with naloxone (Suboxone ${ }^{\circledR}$ ) as an alternative to methadone.

\section{Literature Review}

A literature review was undertaken to determine the evidence-based support for the uses of opioid antagonists and partial agonists/antagonists in abuse deterrent strategy regimens to prevent opioid tolerance and the development of dependence, as well as in the management of opioid detoxification and treatment of withdrawal. All English language randomized controlled trials, observational trials, systematic reviews, and meta-analysis with more than 6 human subjects involving medications/therapies approved for use in the United States published between 1992 and 2007 were included. Studies describing treatment of opioid-induced constipation were excluded because this therapy was not approved for use in the United States at the time of this writing. Interested readers are referred to the systematic review on this subject by Becker et al (3), and the review of opioid complications in this issue. Studies describing treatment of opioid withdrawal other than with agonist/antagonists or antagonists were also excluded as being beyond the focus of this review. 
Table 1. Summary of studies meeting inclusion criteria.

\begin{tabular}{|c|c|c|c|c|c|}
\hline STUDY & PARTICIPANTS & INTERVENTION & OUTCOMES & RESULTS & CONCLUSION \\
\hline $\begin{array}{l}\text { Gowing et al } \\
(18) \\
\text { systematic } \\
\text { review }\end{array}$ & $\begin{array}{l}9 \text { studies }(5 \\
\text { randomized control } \\
\text { trials) } \\
\mathrm{N}=775 \text { participants } \\
\text { were primarily opioid } \\
\text { dependent }\end{array}$ & $\begin{array}{l}\text { Experimental } \\
\text { interventions: } \\
\text { opioid antagonists } \\
\text { in combination with } \\
\text { minimal sedation to } \\
\text { mange withdrawal, } \\
\text { comparison } \\
\text { interventions: other } \\
\text { approaches or different } \\
\text { opioid antagonist regime }\end{array}$ & $\begin{array}{l}\text { Intensity of } \\
\text { withdrawal, } \\
\text { Duration of } \\
\text { treatment/ } \\
\text { treatment } \\
\text { retention, } \\
\text { Adverse effects, } \\
\text { Treatment } \\
\text { completion } \\
\text { Engagement in } \\
\text { further treatment }\end{array}$ & $\begin{array}{l}\text { Withdrawal induced } \\
\text { by opioid antagonists } \\
\text { in combination with } \\
\text { adrenergic agonists is more } \\
\text { intense than withdrawal } \\
\text { managed with clonidine or } \\
\text { lofexidine alone. } \\
\text { Antagonist induced } \\
\text { withdrawal may be more } \\
\text { severe with methadone } \\
\text { rather than a short acting } \\
\text { opioid e.g. heroin. } \\
\text { No significant difference } \\
\text { in rates of treatment } \\
\text { completion for withdrawal } \\
\text { induced by opioid } \\
\text { antagonists + adrenergic } \\
\text { agonist, compared with } \\
\text { adrenergic agonist alone. }\end{array}$ & $\begin{array}{l}\text { Use of opioid } \\
\text { antagonists combined } \\
\text { with adrenergic } \\
\text { agonists is a feasible } \\
\text { approach to manage } \\
\text { opioid withdrawal. } \\
\text { It is unclear if } \\
\text { this approach } \\
\text { reduced duration } \\
\text { of withdrawal or } \\
\text { facilitates transfer to } \\
\text { naltrexone treatment } \\
\text { to a greater extent } \\
\text { than withdrawal } \\
\text { managed primarily } \\
\text { with adrenergic } \\
\text { agonist. }\end{array}$ \\
\hline $\begin{array}{l}\text { Sullivan } \\
\text { et al (19) } \\
\text { prospective, } \\
\text { cross sectional } \\
\text { \& longitudinal } \\
\text { analysis }\end{array}$ & $\begin{array}{l}\text { Patients in } 2 \text { settings: } \\
\text { PCC: }(\mathrm{N}=96) \\
\text { Buprenorphine trial in } \\
\text { primary care clinic } \\
\text { OTP: }(\mathrm{N}=94) \\
\text { methadone } \\
\text { maintenance in local } \\
\text { opioid treatment } \\
\text { Program }\end{array}$ & $\begin{array}{l}\text { Compare PCC subjects } \\
\text { with OTP subjects } \\
\text { Compare PCC patients } \\
\text { with no history of } \\
\text { methadone treatment } \\
\text { (new-to-treatment) } \\
\text { to those with prior } \\
\text { methadone treatment }\end{array}$ & $\begin{array}{l}\begin{array}{l}\text { Clinical } \\
\text { characteristics } \\
\text { of PCC vs. OTP } \\
\text { subjects }\end{array} \\
\text { Characteristics } \\
\text { and treatment } \\
\text { outcomes of } \\
\text { new-to-treatment } \\
\text { PCC vs. with } \\
\text { prior methadone } \\
\text { treatment PCC } \\
\text { subjects }\end{array}$ & $\begin{array}{l}\text { PCC subjects were more } \\
\text { likely to be male, full time } \\
\text { employed, no history of } \\
\text { methadone treatment, } \\
\text { fewer years of opioid } \\
\text { dependence, lower rates of } \\
\text { injection drug use (IDU). } \\
\\
\text { New-to-treatment PCC } \\
\text { subjects were younger, } \\
\text { more likely to be white, } \\
\text { fewer years of opioid } \\
\text { dependence, less likely } \\
\text { to have IDU history and } \\
\text { lower rates of hepatitis C. } \\
\text { Abstinence and treatment } \\
\text { retention were comparable } \\
\text { in both groups. }\end{array}$ & $\begin{array}{l}\text { Office based } \\
\text { treatment of opioid } \\
\text { dependence is } \\
\text { associated with new } \\
\text { types of patients } \\
\text { entering into the } \\
\text { treatment. } \\
\text { Treatment outcomes } \\
\text { with buprenorphine } \\
\text { in a PCC do not vary } \\
\text { based on history of } \\
\text { prior methadone } \\
\text { treatment. }\end{array}$ \\
\hline $\begin{array}{l}\text { Kakko et al } \\
(14) \\
\text { randomized, } \\
\text { placebo } \\
\text { controlled trial }\end{array}$ & $\begin{array}{l}40 \text { heroin dependent } \\
\text { adults, meeting } \\
\text { DSM-IV criteria for } \\
\text { opioid dependence } \\
\text { for at least } 1 \text { year, but } \\
\text { did not fulfill Swedish } \\
\text { criteria for methadone } \\
\text { maintenance treatment }\end{array}$ & $\begin{array}{l}2 \text { groups of 20: } \\
\text { Daily fixed dose SL } \\
\text { buprenorphine } 16 \mathrm{mg} \text { for } \\
12 \text { months } \\
\text { Tapered } 6 \text { day } \\
\text { buprenorphine, followed } \\
\text { by placebo } \\
\text { Individual counseling } \\
\text { weekly and cognitive } \\
\text { behavioral group therapy } \\
\text { to both groups }\end{array}$ & $\begin{array}{l}1 \text { year retention in } \\
\text { treatment } \\
\text { Addiction severity } \\
\text { index (ASI) } \\
\text { 3/week supervised } \\
\text { urine samples }\end{array}$ & $\begin{array}{l}\text { 1-year retention in } \\
\text { treatment was } 75 \% \\
(15 / 20) \text { and } 0 \% \text { in the } \\
\text { buprenorphine and placebo } \\
\text { groups, respectively } \\
(p=0.0001 \text {; risk ratio } 58.7 \\
\text { [95\% CI 7.4-476.4]). } \\
\text { Urine screens were about } \\
75 \% \text { negative for illicit } \\
\text { opiates, central stimulants, } \\
\text { cannabinoids, and } \\
\text { benzodiazepines in patients } \\
\text { remaining in treatment. }\end{array}$ & $\begin{array}{l}\text { Combination of } \\
\text { buprenorphine and } \\
\text { intensive psychosocial } \\
\text { treatment is safe and } \\
\text { highly effective for } \\
\text { heroin dependent } \\
\text { patients. }\end{array}$ \\
\hline $\begin{array}{l}\text { Comer et al (5) } \\
\text { randomized, } \\
\text { double-blind, } \\
\text { placebo } \\
\text { controlled trial }\end{array}$ & $\begin{array}{l}60 \text { heroin-dependent } \\
\text { adults, stratified by sex } \\
\text { and years of heroin use }\end{array}$ & $\begin{array}{l}\text { Received injections of } \\
\text { either placebo or } 192 \\
\text { mg or } 384 \mathrm{mg} \text { of depot } \\
\text { naltrexone }\end{array}$ & $\begin{array}{l}\text { Retention in } \\
\text { treatment and \% } \\
\text { of opioid negative } \\
\text { urine samples }\end{array}$ & $\begin{array}{l}\text { Retention was dose- } \\
\text { related; } 39 \%, 60 \% \text {, and } 68 \% \\
\text { in the placebo, } 192 \text { mg and } \\
384 \text { mg naltrexone groups, } \\
\text { respectively, being retained } \\
\text { in treatment; no significant } \\
\text { difference in \% opioid } \\
\text { negative urine samples } \\
\text { between the placebo and } \\
\text { treatment groups. }\end{array}$ & $\begin{array}{l}\text { Depot naltrexone is } \\
\text { safe and effective in } \\
\text { retaining heroin- } \\
\text { dependent patients in } \\
\text { treatment. }\end{array}$ \\
\hline
\end{tabular}


Table 1.cont.

\begin{tabular}{|c|c|c|c|c|c|}
\hline STUDY & PARTICIPANTS & INTERVENTION & OUTCOMES & RESULTS & CONCLUSION \\
\hline $\begin{array}{l}\text { Johnson et al } \\
\text { (9) } \\
\text { randomized } \\
\text { control trial }\end{array}$ & $\begin{array}{l}220 \text { patients with } \\
\text { opioid dependence } \\
\text { according to DSM-IV } \\
\text { criteria }\end{array}$ & $\begin{array}{l}4 \text { treatment groups: } \\
{ }^{*} \text { Methadone } 20 \mathrm{mg} \text { daily } \\
\text { (control) } \\
{ }^{*} \text { Levomethadyl } 75 \text { - } \\
115 \mathrm{mg} 3 \text { times/week } \\
{ }^{*} \text { Buprenorphine } 16-32 \\
\text { mg } 3 \text { times/ week } \\
{ }^{*} \text { Methadone } 60-100 \mathrm{mg} \\
\text { daily }\end{array}$ & $\begin{array}{l}\text { Retention } \\
\text { in the study; } \\
\text { continued opioid } \\
\text { usage; degree } \\
\text { of continuous } \\
\text { abstinence from } \\
\text { opioid use; and, } \\
\text { patient's global } \\
\text { ratings of severity } \\
\text { of their drug } \\
\text { problem }\end{array}$ & $\begin{array}{l}\text { Greater retention with } \\
\text { high-dose methadone, } \\
\text { buprenorphine and } \\
\text { levomethadyl than control } \\
\text { group. Levomethadyl, } \\
\text { buprenorphine, and high- } \\
\text { dose methadone groups had } \\
\text { fewer opioid-positive urine } \\
\text { specimens than the control } \\
\text { group. Control group gave } \\
\text { the highest severity rating to } \\
\text { their drug problem }\end{array}$ & $\begin{array}{l}\text { Levomethadyl, } \\
\text { buprenorphine, } \\
\text { and high-dose } \\
\text { methadone are more } \\
\text { effective than low- } \\
\text { dose methadone in } \\
\text { reducing illicit opioid } \\
\text { use. }\end{array}$ \\
\hline $\begin{array}{l}\text { Marsch et al } \\
\text { (16) } \\
\text { randomized, } \\
\text { double-blind, } \\
\text { controlled trial }\end{array}$ & $\begin{array}{l}36 \text { self-referred } \\
\text { adolescents (ages } \\
13-18 \text { years) meeting } \\
\text { DMS-IV criteria for } \\
\text { opioid dependence } \\
\text { randomized into a } \\
28 \text { day outpatient } \\
\text { medication-assisted } \\
\text { withdrawal treatment }\end{array}$ & $\begin{array}{l}2 \text { detoxification groups: } \\
{ }^{*} \text { Buprenorphine- flexible } \\
\text { dosing based on weight and } \\
\text { self-reported opioid use } \\
{ }^{*} \text { Clonidine- } 0.1 \text { and } 0.2 \\
\text { mg patches } \\
\text { Behavioral counseling and } \\
\text { incentive contingencies } \\
\text { for both groups }\end{array}$ & $\begin{array}{l}\text { Treatment } \\
\text { retention; opioid } \\
\text { abstinence; HIV } \\
\text { risk behavior }\end{array}$ & $\begin{array}{l}72 \% \text { of patients receiving } \\
\text { buprenorphine retained } \\
\text { in treatment compared to } \\
39 \% \text { receiving clonidine; } \\
64 \% \text { opioid negative urine } \\
\text { tests in the buprenorphine } \\
\text { group compared to } 32 \% \text { in } \\
\text { the clonidine group. HIV } \\
\text { risk behavior significantly } \\
\text { decreased in both groups. }\end{array}$ & $\begin{array}{l}\text { Combining } \\
\text { behavioral } \\
\text { interventions with } \\
\text { buprenorphine is } \\
\text { significantly more } \\
\text { efficacious in treating } \\
\text { opioid-dependent } \\
\text { adolescents than with } \\
\text { clonidine. }\end{array}$ \\
\hline $\begin{array}{l}\text { Ling et al (8) } \\
\text { randomized, } \\
\text { double-blind } \\
\text { controlled trial }\end{array}$ & $\begin{array}{l}736 \text { patients meeting } \\
\text { DSM-iV criteria for } \\
\text { opioid dependence } \\
\text { enrolled at } 12 \text { clinic } \\
\text { sites, for a } 16 \text {-week } \\
\text { maintenance treatment } \\
\text { period }\end{array}$ & $\begin{array}{l}\text { Randomized to } 4 \\
\text { dosage groups and } \\
\text { treated double blind: } \\
1,4,8 \text {, and } 16-\mathrm{mg} / \mathrm{day} \\
\text { buprenorphine } \\
\text { Weekly counseling } \\
\text { sessions for all groups }\end{array}$ & $\begin{array}{l}4 \text { efficacy } \\
\text { domains: } \\
\text { Treatment } \\
\text { retention; illicit } \\
\text { opioid use; opioid } \\
\text { craving; global } \\
\text { ratings of drug } \\
\text { problem severity } \\
\text { by patients and } \\
\text { staff }\end{array}$ & $\begin{array}{l}51 \% \text { of the patients completed } \\
\text { the } 16 \text {-week study. } \\
1 \text { mg buprenorphine group } \\
\text { had significantly worse } \\
\text { retention }(40 \%) \text { compared } \\
\text { to the } 8 \text { mg group }(52 \%) \text {. } \\
1 \mathrm{mg} \text { group had a } \\
\text { significantly lower number of } \\
\text { opioid negative urines than } \\
\text { the other dosage groups. } \\
\text { Significantly higher heroin } \\
\text { graving scale scores were } \\
\text { noted in the } 1 \text { mg group. } \\
\text { Significantly better global } \\
\text { rating scores were obtained } \\
\text { in the } 8 \text { mg group compared } \\
\text { to the } 1 \text { mg group. }\end{array}$ & $\begin{array}{l}\text { 8-mg/day } \\
\text { buprenorphine was } \\
\text { safe, effective, and } \\
\text { superior to } 1 \mathrm{mg} / \text { day } \\
\text { dose in each of the } \\
4 \text { efficacy domains } \\
\text { studied. }\end{array}$ \\
\hline $\begin{array}{l}\text { Johnson et } \\
\text { al (7) } \\
\text { randomized, } \\
\text { double-blind } \\
\text { controlled trial }\end{array}$ & $\begin{array}{l}162 \text { volunteer patients } \\
\text { seeking treatment for } \\
\text { opioid dependence }\end{array}$ & $\begin{array}{l}\text { Randomized into } 3 \\
\text { treatment groups: } \\
{ }^{\star} \text { Buprenorphine } 8 \mathrm{mg} / \\
\text { day; } \\
{ }^{*} \text { Methadone } 60 \mathrm{mg} / \text { day; } \\
{ }^{*} \text { Methadone } 20 \mathrm{mg} / \text { day } \\
\\
17 \text { week maintenance } \\
\text { phase followed by a } 8 \\
\text { week detoxification phase }\end{array}$ & $\begin{array}{l}\text { Retention in } \\
\text { treatment; opioid- } \\
\text { negative urine } \\
\text { samples; and } \\
\text { failure to maintain } \\
\text { abstinence }\end{array}$ & $\begin{array}{l}\text { Retention rates were } \\
\text { significantly greater for } \\
\text { buprenorphine }(30 \%) \text {, } \\
\text { methadone } 60 \mathrm{mg} / \text { day } \\
(20 \%) \text { than for methadone } \\
20 \mathrm{mg} / \text { day }(6 \%) \text { for the entire } \\
25 \text { weeks. Buprenorphine } \\
\text { had significantly more } \\
\text { opioid-negative urine } \\
\text { samples (59\%) than either } \\
\text { methadone } 60 \mathrm{mg} / \text { day ( } 43 \%) \\
\text { or methadone } 20 \mathrm{mg} / \text { day } \\
(39 \%) \text {. Failure to maintain } \\
\text { abstinence was significantly } \\
\text { greater for methadone } 20 \mathrm{mg} / \\
\text { day than buprenorphine. }\end{array}$ & $\begin{array}{l}\text { Buprenorphine } \\
\text { was as effective } \\
\text { as methadone } \\
60 \mathrm{mg} / \text { day and both } \\
\text { were superior to } \\
\text { methadone } 20 \mathrm{mg} / \text { day } \\
\text { in treating opioid } \\
\text { dependence. }\end{array}$ \\
\hline
\end{tabular}


Table 1.cont.

\begin{tabular}{|c|c|c|c|c|c|}
\hline STUDY & PARTICIPANTS & INTERVENTION & OUTCOMES & RESULTS & CONCLUSION \\
\hline $\begin{array}{l}\text { Fudala et al } \\
(12) \\
\text { randomized, } \\
\text { placebo- } \\
\text { controlled trial }\end{array}$ & $\begin{array}{l}\text { Patients meeting DSM- } \\
\text { IV criteria for opioid } \\
\text { dependence, seeking } \\
\text { opiate-substitution } \\
\text { pharmacotherapy. } \\
2 \text { study parts: } 326 \\
\text { patients in } 4 \text {-week } \\
\text { double-blind efficacy } \\
\text { trial; } 461 \text { patients in } \\
\text { 48-week open-label } \\
\text { safety assessment study }\end{array}$ & $\begin{array}{l}\text { Double-blind trial, } \\
\text { subjects randomized to: } \\
\text { - Buprenorphine } 16 \mathrm{mg} / \\
\text { day + naloxone } 4 \mathrm{mg} / \mathrm{day} \\
\text { - Buprenorphine } 16 \mathrm{mg} / \\
\text { day } \\
\text { - Placebo } \\
\text { Open-label study, } \\
\text { daily doses up to: } \\
\text { buprenorphine } 24 \mathrm{mg}+ \\
\text { naloxone } 6 \mathrm{mg}\end{array}$ & $\begin{array}{l}\text { Measures of } \\
\text { treatment efficacy: } \\
\text { \% of opioid- } \\
\text { negative urine } \\
\text { samples; self- } \\
\text { reported craving for } \\
\text { opiates. Secondary } \\
\text { measures: subject/ } \\
\text { staff ratings of } \\
\text { overall status; urine } \\
\text { samples negative } \\
\text { for other drugs of } \\
\text { abuse; retention; } \\
\text { rates of adverse } \\
\text { medical events }\end{array}$ & $\begin{array}{l}\text { Double-blind trial: } \\
\text { Opioid-negative urine } \\
\text { samples were higher in the } \\
\text { buprenorphine + naloxone } \\
(17.8 \%) \text { and buprenorphine } \\
(20.7 \%) \text { groups compared } \\
\text { with placebo (5.8\%). } \\
\text { Buprenorphine + naloxone } \\
\text { and buprenorphine alone } \\
\text { groups reported significantly } \\
\text { less opiate craving than } \\
\text { placebo. Rates of adverse } \\
\text { events were similar between } \\
\text { active-treatment and } \\
\text { placebo groups. }\end{array}$ & $\begin{array}{l}\text { Double-blind trial } \\
\text { was terminated early } \\
\text { because buprenorphine } \\
\text { and naloxone in } \\
\text { combination and } \\
\text { buprenorphine alone } \\
\text { were found to have } \\
\text { greater efficacy than } \\
\text { placebo. } \\
\text { Open-label study } \\
\text { showed the } \\
\text { buprenorphine and } \\
\text { naloxone combine } \\
\text { treatment was safe } \\
\text { and well tolerated. }\end{array}$ \\
\hline $\begin{array}{l}\text { Gerra et al (21) } \\
\text { prospective } \\
\text { observational } \\
\text { study }\end{array}$ & $\begin{array}{l}60 \text { naltrexone } \\
\text { treatment-seeking } \\
\text { heroin-dependent } \\
\text { patients, meeting } \\
\text { DSM-IV criteria were } \\
\text { enrolled in a } 12 \text { week } \\
\text { clinical evaluation }\end{array}$ & $\begin{array}{l}\text { Non-randomized into } 2 \\
\text { treatment groups: } \\
\text { Naltrexone 50mg; } \\
\text { Buprenorphine } 4 \mathrm{mg}+ \\
\text { naltrexone } 50 \mathrm{mg}\end{array}$ & $\begin{array}{l}\text { Retention in } \\
\text { treatment; } \\
\text { negative } \\
\text { urinalysis; } \\
\text { changes in } \\
\text { psychological } \\
\text { symptoms; and } \\
\text { craving scores }\end{array}$ & $\begin{array}{l}\text { Retention rate was } \\
\text { significantly higher }(73.3 \%) \\
\text { in the buprenorphine + } \\
\text { naltrexone group compared } \\
\text { with the naltrexone alone } \\
(40 \%) \text {. Buprenorphine }+ \\
\text { naltrexone group showed } \\
\text { a significantly lower rate } \\
\text { of positive urinalysis } \\
\text { for opiates and cocaine. } \\
\text { Psychological symptoms } \\
\text { and craving scores decreased } \\
\text { significantly in the } \\
\text { buprenorphine + naltrexone } \\
\text { group, as compared with } \\
\text { naltrexone alone. }\end{array}$ & $\begin{array}{l}\text { Combination of } \\
\text { buprenorphine } \\
\text { and naltrexone } \\
\text { significantly } \\
\text { improved the } \\
\text { treatment outcome } \\
\text { of opioid dependence } \\
\text { over the use of opioid } \\
\text { antagonists alone. }\end{array}$ \\
\hline $\begin{array}{l}\text { Chindalore et } \\
\text { al }(4) \\
\text { randomized, } \\
\text { double-blind, } \\
\text { placebo- } \\
\text { controlled trial }\end{array}$ & $\begin{array}{l}360 \text { patients with } \\
\text { moderate to severe } \\
\text { chronic osteoarthritis } \\
\text { pain involving hip and } \\
\text { knees were enrolled in } \\
\text { a 3-week, Phase II trial } \\
\text { assessing the safety and } \\
\text { efficacy of Oxytrex }\end{array}$ & $\begin{array}{l}\text { Randomized into } 4 \\
\text { treatment groups: } \\
\text { - Placebo QID } \\
\text { - Oxycodone QID } \\
\text { - Oxytrex QID } \\
\text { - Oxytrex BID } \\
\text { Active-treatment doses } \\
\text { of oxycodone escalated } \\
\text { as the same total daily } \\
\text { dose from } 10 \mathrm{mg} / \text { day to } \\
40 \mathrm{mg} / \text { day. Oxytrex was } \\
\text { formulated to contain } \\
0.001 \mathrm{mg} \text { naltrexone per } \\
\text { dose. }\end{array}$ & $\begin{array}{l}\text { Daily numerical } \\
\text { pain rating; } \\
\text { weekly evaluations } \\
\text { of the quality } \\
\text { of analgesia; } \\
\text { duration and } \\
\text { extent of pain } \\
\text { control; global } \\
\text { assessment of the } \\
\text { study drug; SF-12 } \\
\text { Health Survey; } \\
\text { and WOMAC } \\
\text { Osteoarthritis } \\
\text { Index }\end{array}$ & $\begin{array}{l}32.2 \% \text { of the patients did } \\
\text { not complete the study. } \\
\text { Oxytrex bid produced a } 39 \% \\
\text { reduction in pain intensity, } \\
\text { significantly greater than } \\
\text { placebo or the other } 3 \\
\text { treatment groups. Oxytrex } \\
\text { bid was superior to placebo } \\
\text { and the other active treatment } \\
\text { groups in the quality of } \\
\text { analgesia, duration of pain } \\
\text { control, global assessment, } \\
\text { and WOMAC total score. } \\
\text { Incidence of side effects was } \\
\text { comparable between active- } \\
\text { treatment groups. }\end{array}$ & $\begin{array}{l}\text { Oxytrex bid provided } \\
\text { greater efficacy in } \\
\text { pain control and } \\
\text { quality of analgesia } \\
\text { at a lesser dosing } \\
\text { frequency. }\end{array}$ \\
\hline $\begin{array}{l}\text { Tamaskar et } \\
\text { al (2) } \\
\text { Retrospective } \\
\text { observational } \\
\text { study }\end{array}$ & $\begin{array}{l}\text { Charts from } 64 \text { patients } \\
\text { with mild to moderate } \\
\text { heroin addiction, who } \\
\text { underwent opioid } \\
\text { detoxification were } \\
\text { reviewed }\end{array}$ & $\begin{array}{l}\text { Patients were categorized } \\
\text { into } 2 \text { treatment groups: } \\
{ }^{*} \text { Oral tramadol } \\
{ }^{*} \text { SQ buprenorphine } \\
\text { Clonidine as needed for } \\
\text { breakthrough withdrawal }\end{array}$ & $\begin{array}{l}\text { Severity of opioid } \\
\text { withdrawal- } \\
\text { average Clinical } \\
\text { Institute Narcotic } \\
\text { Assessment } \\
\text { (CINA); length of } \\
\text { stay; amount of } \\
\text { clonidine needed } \\
\text { for withdrawal } \\
\text { control. }\end{array}$ & $\begin{array}{l}\text { Average CINA maximum } \\
\text { for tramadol was } 9 \text { vs. } 11.2 \\
\text { for buprenorphine. Length } \\
\text { of stay was } 3.7 \text { days with } \\
\text { tramadol vs. } 4.1 \text { days with } \\
\text { buprenorphine. Use of } \\
\text { clonidine pills per patient } \\
\text { was } 1.6 \text { with tramadol vs. } \\
0.1 \text { with buprenorphine. } \\
\text { Leaving against medical } \\
\text { advice was higher for the } \\
\text { buprenorphine group. }\end{array}$ & $\begin{array}{l}\text { Tramadol compared } \\
\text { favorably to } \\
\text { buprenorphine in the } \\
\text { management of acute } \\
\text { withdrawal from less } \\
\text { than } 10 \text { bags per day } \\
\text { of heroin. }\end{array}$ \\
\hline
\end{tabular}


Table 1.cont.

\begin{tabular}{|c|c|c|c|c|c|}
\hline STUDY & PARTICIPANTS & INTERVENTION & OUTCOMES & RESULTS & CONCLUSION \\
\hline $\begin{array}{l}\text { Mitchell et } \\
\text { al (6) } \\
\text { Prospective } \\
\text { observational, } \\
\text { crossover study }\end{array}$ & $\begin{array}{l}18 \text { methadone } \\
\text { maintenance patients }\end{array}$ & $\begin{array}{l}\text { Subjects were } \\
\text { transitioned from oral } \\
\text { methadone to a once- } \\
\text { daily slow-release oral } \\
\text { morphine (SROM) } \\
\text { product for a 6-week } \\
\text { period; then methadone } \\
\text { maintenance was } \\
\text { recommenced. }\end{array}$ & $\begin{array}{l}\text { Opioid } \\
\text { withdrawal } \\
\text { severity during } \\
\text { transition; } 4 \text { weeks } \\
\text { after transition, } \\
\text { outcome } \\
\text { assessments made } \\
\text { for treatment } \\
\text { preference, } \\
\text { efficacy, } \\
\text { acceptability, } \\
\text { health, depression } \\
\text { and sleep. }\end{array}$ & $\begin{array}{l}\text { Withdrawal severity was } \\
\text { significantly greater during } \\
\text { transition to SROM than } \\
\text { during resumption of } \\
\text { methadone maintenance. } \\
78 \% \text { preferred SROM over } \\
\text { methadone } 22 \% \text {. SROM was } \\
\text { associated with significantly } \\
\text { better outcomes over } \\
\text { methadone in treatment } \\
\text { efficacy, acceptability, fewer } \\
\text { side effects and social } \\
\text { functioning. }\end{array}$ & $\begin{array}{l}\text { SROM could serve } \\
\text { as an effective } \\
\text { maintenance } \\
\text { pharmacotherapy for } \\
\text { opioid dependence. }\end{array}$ \\
\hline $\begin{array}{l}\text { Correia et al } \\
(20) \\
\text { Prospective } \\
\text { observational } \\
\text { study }\end{array}$ & $\begin{array}{l}8 \text { patients with active } \\
\text { opioid dependence } \\
\text { according to DSM-IV } \\
\text { criteria }\end{array}$ & $\begin{array}{l}\text { Each subject maintained } \\
\text { on } 3 \text { different } \\
\text { double-blind doses } \\
\text { of buprenorphine/ } \\
\text { naloxone }(\mathrm{B} / \mathrm{N}): 8 / 2, \\
16 / 4 \text { and } 32 / 8 \mathrm{mg} \text {; } \\
\text { order of administration } \\
\text { was randomized. } \\
\text { Challenge doses of IM } \\
\text { hydromorphone } 0,6 \text { and } \\
12 \mathrm{mg} \text {, given double- } \\
\text { blind, at } 2,26,50,74 \text { and } \\
98 \text { hours after last dose } \\
\text { of } \mathrm{B} / \mathrm{N} \text {. }\end{array}$ & $\begin{array}{l}\text { Efficacy of opioid } \\
\text { blockade and } \\
\text { spontaneous } \\
\text { withdrawal assessed } \\
\text { using: physiologic } \\
\text { parameters; subject } \\
\text { VAS scores and } \\
\text { subject/observer } \\
\text { adjective-rating } \\
\text { questionnaires; } \\
\text { psychomotor/ } \\
\text { cognitive } \\
\text { performance } \\
\text { measures. }\end{array}$ & $\begin{array}{l}\text { As the time period } \\
\text { increased since the last } \\
\text { B/N dose, hydromorphone } \\
\text { challenge doses produced } \\
\text { decreased reports of } \\
\text { opioid agonist effects } \\
\text { and increases in mild } \\
\text { withdrawal symptoms. } \\
\text { Substantial but incomplete } \\
\text { blockade against opioid } \\
\text { effect was provided by B/N } \\
\text { for } 98 \text { hours. }\end{array}$ & $\begin{array}{l}\mathrm{B} / \mathrm{N} \text { doses greater } \\
\text { than } 8 / 2 \mathrm{mg} \\
\text { provide minimal } \\
\text { benefit in terms of } \\
\text { opioid blockade } \\
\text { and withdrawal } \\
\text { suppression and } \\
\text { intermittent } \\
\mathrm{B} / \mathrm{N} \text { dosing may be } \\
\text { effective for up to } 98 \\
\text { hours. }\end{array}$ \\
\hline $\begin{array}{l}\text { Ahmadi et al } \\
(11) \\
\text { Randomized } \\
\text { controlled trial }\end{array}$ & $\begin{array}{l}204 \text { IV buprenorphine- } \\
\text { dependent patients, for } \\
\text { at least } 6 \text { mos., seeking } \\
\text { treatment, meeting } \\
\text { DMS-IV criteria for } \\
\text { opioid dependence }\end{array}$ & $\begin{array}{l}\text { Randomized into } 3 \\
\text { treatment groups: } \\
\text { 1. Oral methadone } 50 \mathrm{mg} \\
\text { 2. SL buprenorphine } 5 \mathrm{mg} \\
\text { 3. Oral naltrexone } 50 \mathrm{mg}\end{array}$ & $\begin{array}{l}\text { Treatment } \\
\text { retention }\end{array}$ & $\begin{array}{l}\text { Overall, } 54.4 \% \text { completed } \\
\text { the } 24 \text {-week study. } \\
83.8 \% \text { in methadone group } \\
58.8 \% \text { in buprenorphine } \\
\text { group } \\
20.6 \% \text { in naltrexone group }\end{array}$ & $\begin{array}{l}\text { Methadone and } \\
\text { buprenorphine } \\
\text { are effective for } \\
\text { maintenance treatment } \\
\text { of buprenorphine- } \\
\text { dependent patients. } \\
\end{array}$ \\
\hline $\begin{array}{l}\text { Lintzeris et al } \\
(10) \\
\text { Prospective } \\
\text { randomized } \\
\text { controlled trial }\end{array}$ & $\begin{array}{l}114 \text { heroin-dependent } \\
\text { patients, meeting } \\
\text { DSM-IV criteria, } \\
\text { seeking ambulatory } \\
\text { withdrawal treatment, } \\
\text { 8-day program with } \\
\text { 4-week follow-up }\end{array}$ & $\begin{array}{l}\text { Randomized into } 2 \\
\text { treatment groups: } \\
\text { Control group } \\
\text { receiving clonidine } \\
\text { and other symptomatic } \\
\text { medications; } \\
\text { Experimental group SL } \\
\text { buprenorphine regimen } \\
\text { Open-label study }\end{array}$ & $\begin{array}{l}\text { Self-reported } \\
\text { heroin use during } \\
\text { withdrawal; } \\
\text { retention in } \\
\text { withdrawal } \\
\text { treatment; } \\
\text { participation in } \\
\text { post withdrawal } \\
\text { treatment; severity } \\
\text { of withdrawal; } \\
\text { adverse effects. }\end{array}$ & $\begin{array}{l}\text { Significantly more } \\
\text { experimental participants } \\
\text { reported no heroin use } \\
\text { during withdrawal. } 86 \% \\
\text { experimental participants } \\
\text { compared to } 57 \% \text { of } \\
\text { controls completed the } \\
\text { withdrawal program. } \\
62 \% \text { of experimental } \\
\text { participants compared } \\
\text { to } 39 \% \text { of controls were } \\
\text { retained in some form of } \\
\text { treatment post withdrawal. } \\
\text { Experimental group } \\
\text { reported significantly less } \\
\text { withdrawal severity. }\end{array}$ & $\begin{array}{l}\text { Buprenorphine } \\
\text { is more effective } \\
\text { than clonidine } \\
\text { and symptomatic } \\
\text { medications in } \\
\text { treating short-term } \\
\text { ambulatory heroin } \\
\text { withdrawal, with } \\
\text { greater retention, } \\
\text { less heroin use, less } \\
\text { withdrawal severity } \\
\text { and increased post } \\
\text { withdrawal treatment } \\
\text { retention. }\end{array}$ \\
\hline $\begin{array}{l}\text { Ling et al (15) } \\
\text { randomized } \\
\text { controlled trial }\end{array}$ & $\begin{array}{l}344 \text { participants-113 } \\
\text { in-patient and } 231 \text { out- } \\
\text { patient, meeting DSM- } \\
\text { IV criteria for opioid } \\
\text { dependence, seeking } \\
\text { treatment for opioid } \\
\text { withdrawal, in a 13-day } \\
\text { detoxification study }\end{array}$ & $\begin{array}{l}\text { In-patients and } \\
\text { out-patients were } \\
\text { randomized into } 2 \\
\text { treatment groups on a } \\
2: 1 \text { ratio buprenorphine- } \\
\text { naloxone (bup-nx): } \\
\text { clonidine groups }\end{array}$ & $\begin{array}{l}\text { Treatment } \\
\text { retention, negative } \\
\text { urine samples, } \\
\text { withdrawal } \\
\text { severity, opioid } \\
\text { craving }\end{array}$ & $\begin{array}{l}\text { Both inpatient and } \\
\text { outpatient bup-nx } \\
\text { groups had significantly } \\
\text { better retention, fewer } \\
\text { withdrawal symptoms, and } \\
\text { significantly lower craving } \\
\text { rating than the clonidine } \\
\text { groups. }\end{array}$ & $\begin{array}{l}\text { Buprenorphine- } \\
\text { naloxone is clinically } \\
\text { more effective than } \\
\text { clonidine in treating } \\
\text { opioid detoxification. }\end{array}$ \\
\hline
\end{tabular}


Table 1.cont.

\begin{tabular}{|c|c|c|c|c|c|}
\hline STUDY & PARTICIPANTS & INTERVENTION & OUTCOMES & RESULTS & CONCLUSION \\
\hline $\begin{array}{l}\text { Mattick et al } \\
\text { (13) } \\
\text { randomized } \\
\text { double-blind } \\
\text { controlled trial }\end{array}$ & $\begin{array}{l}405 \text { opioid-dependent } \\
\text { patients, meeting } \\
\text { DSM-IV criteria, } \\
\text { seeking treatment, over } \\
\text { a } 13 \text {-week treatment } \\
\text { period }\end{array}$ & $\begin{array}{l}\text { Randomized into } 2 \\
\text { treatment groups: } \\
\text { SL buprenorphine; } \\
\text { oral methadone } \\
\text { flexible dosing regimen, } \\
\text { double-blind }\end{array}$ & $\begin{array}{l}\text { Efficacy } \\
\text { assessments: } \\
\text { Retention in } \\
\text { treatment; opioid } \\
\text { negative urine } \\
\text { samples; Opiate } \\
\text { Treatment } \\
\text { Index (OTI) } \\
\text { and Symptom } \\
\text { Checklist-90- } \\
\text { R (SCL-90-R) } \\
\text { measures of heroin, } \\
\text { illicit drug use, } \\
\text { HIV-risk behavior, } \\
\text { social functioning, } \\
\text { physical and } \\
\text { mental status }\end{array}$ & $\begin{array}{l}\text { Overall, } 54.8 \% \text { of patients } \\
\text { completed the } 13 \text {-week } \\
\text { trial. } 59 \% \text { of methadone } \\
\text { compared to } 50 \% \text { of } \\
\text { buprenorphine patients } \\
\text { completed the trial. No } \\
\text { significant differences } \\
\text { in opioid negative urine } \\
\text { samples between the } \\
2 \text { groups. Significant } \\
\text { improvements, but no } \\
\text { differences between groups } \\
\text { in self-reported OTI and } \\
\text { SCL-90-R measures. }\end{array}$ & $\begin{array}{l}\text { Both methadone and } \\
\text { buprenorphine are } \\
\text { comparably effective } \\
\text { in treating opioid } \\
\text { dependence. }\end{array}$ \\
\hline $\begin{array}{l}\text { Raistrick et al } \\
(17) \\
\text { randomized } \\
\text { controlled trial }\end{array}$ & $\begin{array}{l}481 \text { heroin users } \\
\text { seeking detoxification } \\
\text { meeting DSM-IV } \\
\text { criteria for opioid- } \\
\text { dependence, first-time } \\
\text { detoxifications }\end{array}$ & $\begin{array}{l}210 \text { participants } \\
\text { randomized into } 2 \\
\text { treatment regimens: } \\
\text { 1. } 5 \text {-day lofexidine } \\
\text { 2. } 7 \text {-day buprenorphine } \\
\text { 271 participants opted to } \\
\text { choose one of the } 2 \\
\text { Open-label }\end{array}$ & $\begin{array}{l}\text { Outcome } \\
\text { measures: } \\
\text { completion of } \\
\text { detoxification } \\
\text { regimen; } \\
\text { abstinence at } \\
\text { 1-month follow- } \\
\text { up; severity of } \\
\text { withdrawal; } \\
\text { treatment } \\
\text { acceptability }\end{array}$ & $\begin{array}{l}65 \% \text { of participants on } \\
\text { buprenorphine compared to } \\
46 \% \text { of those on lofexidine } \\
\text { completed detoxification. } \\
45.9 \% \text { of the buprenorphine } \\
\text { group and } 35.7 \% \text { of the } \\
\text { lofexidine group reported } \\
\text { abstinence at } 1 \text {-month. } \\
\text { Significant improvements, } \\
\text { but no statistically } \\
\text { significant differences } \\
\text { between groups in measures } \\
\text { of social functioning } \\
\text { mental status. Severity of } \\
\text { withdrawal was rated less } \\
\text { with buprenorphine. }\end{array}$ & $\begin{array}{l}\text { Buprenorphine is } \\
\text { at least as effective } \\
\text { as lofexidine } \\
\text { detoxification. }\end{array}$ \\
\hline
\end{tabular}

\section{Results}

Table 1 summarizes the studies considered for this review. All of the studies found, with the exception of Chindalore et al (4), Comer et al (5) and Mitchell et al (6), dealt with the use of buprenorphine for the treatment of opioid addiction. The Chindalore et al (4) study involved the addition of naltrexone to oxycodone to prevent tolerance and hyperalgesia. Since the formulation (Oxytrex®) is not currently available in the United States, it is not further discussed. In like manner, Comer et al (5) documented the efficacy of depot naltrexone for the treatment of opioid addiction. As this treatment is not currently available in the United States, it is not further discussed. Mitchell et al (6) showed that sustained release oral morphine could be substituted for methadone for opioid maintenance therapy.
A total of 17 articles were found evaluating the use of buprenorphine for the treatment of opioid addiction. Of these, 1 was a systematic review, 12 were randomized controlled trials (RCTs), and 4 were observational studies.

Several studies have reported on buprenorphine versus methadone maintenance. Johnson et al (7) in 1992 compared the efficacy of buprenorphine to methadone for short-term maintenance and opioid detoxification. In a randomized, double blind, parallel group study buprenorphine $8 \mathrm{mg} /$ day was compared with methadone $60 \mathrm{mg} /$ day and methadone 20mg/ day. There was a 17-week maintenance phase followed by an 8-week detoxification phase. Retention rates and percentage urine samples negative for opioids was significantly greater for buprenorphine and 
methadone $60 \mathrm{mg} / \mathrm{d}$ than for methadone $20 \mathrm{mg} /$ day during the maintenance phase. There was no difference between groups with respect to negative urine samples for opioids during the detoxification phase. Buprenorphine was considered to be as effective as high dose methadone in reducing illicit opioid use and in maintaining patients in treatment for 25 weeks.

A multicenter randomized, double blind clinical trial by Ling et al (8) evaluated safety and efficacy of different doses of buprenorphine in heroin addicts. Patients received 1, 4, 8 or $16 \mathrm{mg} /$ day of buprenorphine. Outcomes in the $8 \mathrm{mg}$ group were significantly better than in the $1 \mathrm{mg}$ group in all 4 efficacy domains: treatment retention, illicit opioid use, opioid craving, and global ratings by patient and staff; no deaths occurred in either group and the $8 \mathrm{mg}$ group did not show an increase in the frequency of adverse events. The $16 \mathrm{mg}$ group did better than the $8 \mathrm{mg}$ group but the differences did not reach statistical significance. The median percentage of clinics attended by the 375 patients who remained in treatment was $89 \%$ and $18 \%$ (63/375) attended clinics less than $70 \%$ of the time. Missed urine samples was only $18 \%$ among completers. Sixty-eight patients completed 16 weeks of treatment without producing a single urine negative for opioids.

Johnson et al (9) studied the use of illicit opioids in 220 opioid dependent patients randomized to receive either levomethadyl, buprenorphine, high-dose methadone, or low dose methadone. The percentage of patients with 12 or more consecutive opioid-negative urine specimens was $36 \%$ in levomethadyl group, $26 \%$ in buprenorphine group, $28 \%$ in high does methadone group, and $8 \%$ in low dose methadone group. As compared with low dose methadone, participants taking levomethadyl acetate had a higher rate of continuous abstinence from opioids, and those taking buprenorphine and high dose methadone had a trend towards higher rate of continuous abstinence.

Lintzeris et al (10) showed that buprenorphine was more effective than clonidine in treating opioid withdrawal. Ahmadi et al (11) looked at patients who were dependent upon IV buprenorphine and found that oral methadone $20 \mathrm{mg}$ and sublingual buprenorphine $5 \mathrm{mg}$, but not oral naltrexone, provided effective maintenance therapy over 24 weeks. Fudala et al (12) conducted a 4-week, multicenter randomized, placebo controlled trial on 326 opiate dependent patients and compared efficacy of buprenorphine $16 \mathrm{mg}$ alone, buprenorphine $16 \mathrm{mg}$ with naloxone $4 \mathrm{mg}$, and placebo. The trial was stopped early because of the greater efficacy of the buprenorphine treatments. The active treatment groups reported less opiate craving. Safety of buprenorphine and naloxone was assessed from data obtained on 461 opiate addicts in an open label study and showed that the combined treatment was safe and well tolerated.

Mattick et al (13), in 2003, confirmed that both buprenorphine and methadone were effective in treating opioid dependence. Kakko et al (14), in a randomized placebo-controlled trial from Sweden, evaluated the long-term effectiveness of buprenorphine in heroin dependent individuals. One year efficacy of buprenorphine in combination with intensive psychosocial therapy for treatment of heroin dependence was assessed by taking 40 heroin dependent individuals and randomly allocating them either to sublingual buprenorphine $12 \mathrm{mg} /$ day for 12 months or to a tapered 6-day regimen of buprenorphine, followed by placebo. Thrice weekly supervised urine samples were obtained to detect illicit drug use. One year retention in treatment was $75 \%$ in the buprenorphine group and $0 \%$ in the placebo group $(p=0.0001$; risk ratio 58.7 [95\% Cl7.4-467.4]). Urine screens were about $75 \%$ negative for illicit drugs in patients remaining in the treatment.

Ling et al (15) showed in 2005 that the combination of buprenorphine and naloxone in a 2:1 ratio was more effective than clonidine in successfully detoxifying opioid addicts over a 13-day period. Marsch et al (16) in 2005, studied opioid-dependent adolescents and found that buprenorphine coupled with behavioral therapy was more effective in completing withdrawal over a 28-day period than was clonidine coupled with behavioral therapy. Raistrick et al (17) showed that in first-time detoxification from heroin, buprenorphine was at least as effective as lofexidine in successfully detoxifying patients over $5-7$ days.

Gowing et al (18), in 2006, performed a Cochrane Review of the use of opioid antagonists for the treatment of opioid withdrawal, looking at 9 studies, including 5 randomized controlled trials. Gowing et al concluded that the use of opioid antagonists combined with alpha2 adrenergic agonists is a feasible approach to the management of opioid withdrawal.

Four observational studies document the efficacy of buprenorphine. A cross-sectional and longitudinal analysis by Sullivan et al (19) showed that patients in office-based buprenorphine treatments in a primary care clinic (PCC) are different from those enrolled in a local opioid treatment program (OTP) in a methadone 
clinic. PCC subjects compared with OTP subjects were more likely to be male $(77 \%$ versus $55 \%)$, full-time employed ( $46 \%$ versus $15 \%, p<0.001$ ), have no history of prior methadone treatment ( $46 \%$ versus $61 \%$ ), have fewer years of opioid dependence (10 versus $15, p<0.001)$, and lower rates of injection drug use (IDU) (46\% versus $61 \%$ ). Furthermore, within the PCC group, the new-to-treatment subjects were younger, more likely to be white, had fewer years of opioid dependence, less likely to have a history of IDU, and lower rates of hepatitis $C$ than subjects with prior methadone treatment. These results suggest office-based buprenorphine treatment expands access to treatment for patients who may not enroll in methadone clinics, and facilitate earlier access to treatment for patients who have more recently initiated opioid use.

Correia et al (20) showed that there was no minimal benefit in increasing the buprenorphine/naloxone dose above $8 \mathrm{mg} / 2 \mathrm{mg}$ day in terms of response to a hydromorphone challenge, and that an intermittent dose of buprenorphine might last as long as 98 hours. Tamaskar et al (2), in a retrospective study, found that the severity of withdrawal from less than 10 bags of heroin was slightly less with tramadol than with buprenorphine. Gerra et al (21), in a prospective study published in 2005, showed that over a 12-week period, the combination of buprenorphine and naltrexone was significantly more effective in retaining treatment than was naltrexone alone.

\section{Discussion}

Various studies have evaluated efficacy and safety for opioid agonists, partial agonist, and antagonists in treating opioid dependence. The measure used to determine treatment efficacy are treatment retention, illicit opioid use, opioid craving, and global ratings by patient and staff. However, treat- ment retention does not tell the whole story. For example, in the study by Ling et al (15), 18\% attended clinics less than $70 \%$ of the time. Missed clinic visits translate into missing urine samples, which was $18 \%$ among completers. Sixty-eight patients completed 16 weeks of treatment without producing a single urine negative for opioids. Clinic attendance without reduction in opioid use cannot be considered as unqualified therapeutic success for buprenorphine. Acceptance of efficacy of buprenorphine as a maintenance treatment has to be tempered by the reality that drug use status of many patients will not be altered by buprenorphine (15).

Detoxification means assisting street heroin addicts to become abstinent or discontinuing patients from opioid maintenance. An effective medication for detoxification should suppress withdrawal symptoms sufficiently to allow an opiate dependent person to become opiate free. Few studies have examined this aspect of treatment.

\section{Conclusion}

Seventeen studies, 1 systematic review, 12 RCTs, and 4 observational series, which document the efficacy and safety of buprenorphine alone and in combination with naloxone in detoxifying and maintaining abstinence from illicit drugs in patients with opioid addiction, were reviewed.

Additional new uses for antagonists, including their use to prevent tolerance with chronic opioid use, may soon be available.

\section{Acknowledgements}

We are grateful to the editors of Pain Physician for their comprehensive review and constructive criticism and assisting in improvements of this manuscript. 


\section{References}

1. Gal TJ, DiFazio CA, Dixon R. Prolonged blockade of opioid effect with oral naImefene. Clin Pharmacol Ther 1986; 40:537-542.

2. Tamaskar R, Parran TV, Jr, Heggi A, Brateanu A, Rabb M, Yu J. Tramadol versus buprenorphine for the treatment of opiate withdrawal: A retrospective cohort control study. J Addict Dis 2003; 22:512.

3. Becker G, Galandi D, Blum HE. Peripherally acting opioid antagonists in the treatment of opiate-related constipation: A systematic review. J Pain Symptom Manage 2007; 34:547-565.

4. Chindalore VL, Craven RA, Yu KP, Butera PG, Burns LH, Friedmann N. Adding ultralow-dose naltrexone to oxycodone enhances and prolongs analgesia: A randomized, controlled trial of Oxytrex. J Pain 2005; 6:392-399.

5. Comer SD, Sullivan MA, Yu E, Rothenberg JL, Kleber HD, Kampman K, Dackis C, O'Brien CP. Injectable, sustainedrelease naltrexone for the treatment of opioid dependence: A randomized, placebo-controlled trial. Arch Gen Psychiatry 2006; 63:210-218.

6. Mitchell TB, White JM, Somogyi AA, Bochner F. Slow-release oral morphine versus methadone: A crossover comparison of patient outcomes and acceptability as maintenance pharmacotherapies for opioid dependence. Addiction 2004; 99:940-945.

7. Johnson RE, Jaffe JH, Fudala PJ. A controlled trial of buprenorphine treatment for opioid dependence. JAMA 1992; 267:2750-2755.

8. Ling W, Charuvastra C, Collins JF, Batki S, Brown LS, Jr, Kintaudi P, Wesson DR, McNicholas L, Tusel DJ, Malkerneker U, Renner JA, Jr, Santos E, Casadonte P, Fye C, Stine S, Wang RI, Segal D. Buprenorphine maintenance treatment of opiate dependence: A multicenter, randomized clinical trial. Addiction 1998; 93:475-486.

9. Johnson RE, Chutuape MA, Strain EC, Walsh SL, Stitzer ML, Bigelow GE. A comparison of levomethadyl acetate, buprenorphine, and methadone for opioid dependence. N Engl / Med 2000; 343:1290-1297.

10. Lintzeris N, Bell J, Bammer G, Jolley DJ, Rushworth L. A randomized controlled trial of buprenorphine in the management of short-term ambulatory heroin withdrawal. Addiction 2002; 97:13951404.

11. Ahmadi J, Ahmadi K, Ohaeri J. Controlled, randomized trial in maintenance treatment of intravenous buprenorphine dependence with naltrexone, methadone or buprenorphine: A novel study. Eur J Clin Invest 2003; 33:824-829.

12. Fudala PJ, Bridge TP, Herbert S, Williford WO, Chiang CN, Jones K, Collins J, Raisch D, Casadonte P, Goldsmith RJ, Ling W, Malkerneker U, McNicholas L, Renner J, Stine S, Tusel D. Office-based treatment of opiate addiction with a sublingual-tablet formulation of buprenorphine and naloxone. N Engl J Med 2003; 349:949-958.

13. Mattick RP, Ali R, White JM, O'Brien S, Wolk S, Danz C. Buprenorphine versus methadone maintenance therapy: A randomized double-blind trial with 405 opioid-dependent patients. Addiction 2003; 98:441-452.

14. Kakko J, Svanborg KD, Kreek MJ, Heilig M. 1-year retention and social function after buprenorphine-assisted relapse prevention treatment for heroin dependence in Sweden: A randomised, placebo-controlled trial. Lancet 2003; 361:662-668.

15. Ling W, Amass L, Shoptaw S, Annon
JJ, Hillhouse M, Babcock D, Brigham G, Harrer J, Reid M, Muir J, Buchan B, Orr D, Woody G, Krejci J, Ziedonis D. A multi-center randomized trial of buprenorphine-naloxone versus clonidine for opioid detoxification: Findings from the National Institute on Drug Abuse Clinical Trials Network. Addiction 2005; 100:1090-1100.

16. Marsch LA, Bickel WK, Badger GJ, Stothart ME, Quesnel KJ, Stanger C, Brooklyn J. Comparison of pharmacological treatments for opioid-dependent adolescents: A randomized controlled trial. Arch Gen Psychiatry 2005; 62:11571164.

17. Raistrick $D$, West $D$, Finnegan 0 , Thistlethwaite G, Brearley R, Banbery J. A comparison of buprenorphine and lofexidine for community opiate detoxification: Results from a randomized controlled trial. Addiction 2005; 100: 18601867.

18. Gowing L, Ali R, White J. Opioid antagonists with minimal sedation for opioid withdrawal. Cochrane Database Syst Rev 2006; 1:CDoo2021.

19. Sullivan LE, Chawarski M, O'Connor PG, Schottenfeld RS, Fiellin DA. The practice of office-based buprenorphine treatment of opioid dependence: Is it associated with new patients entering into treatment? Drug Alcohol Depend 2005; 79:113-116.

20. Correia CJ, Walsh SL, Bigelow GE, Strain EC. Effects associated with doubleblind omission of buprenorphine/naloxone over a 98-h period. Psychopharmacology (Berl) 2006; 189:297-306.

21. Gerra G, Fantoma A, Zaimovic A. NaItrexone and buprenorphine combination in the treatment of opioid dependence. I Psychopharmacol 2006; 20:806-814. 